\title{
O USO DE ÓXIDO DE GRAFENO PARA REPARAÇÃO DE PAVIMENTO ASFÁLTICO - UMA REVISÃO
}

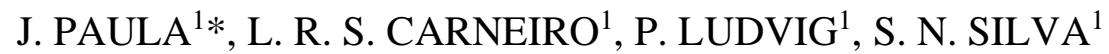 \\ ${ }^{1}$ Departamento de Engenharia Civil, CEFET-MG, Av. Amazonas, 7675, Belo Horizonte - MG - Brasil
}

\section{RESUMO}

O modal rodoviário é o principal sistema logístico do Brasil, sendo responsável pelo transporte de $60 \%$ de todas as cargas no território e $57 \%$ das rodovias brasileiras apresentam algum tipo de manifestação patológica, isso ocorre devido a projetos de design ruins, aumento imprevisto de cargas rodoviárias e falta de manutenção preventiva. Estudos recentes mostraram o potencial da adição de óxido de grafeno (GO) em revestimentos asfálticos. Este trabalho tem como objetivo discutir o papel do óxido de grafeno na melhoria das propriedades dos revestimentos asfálticos e nos microrrevestimentos asfálticos. De acordo com a literatura, a introdução de $3 \%$ de óxido de grafeno melhora a viscosidade, a temperatura de trabalho e a resistência aos ciclos de fadiga.

Palavras-chave: óxido de grafeno; revestimentos asfálticos; micro revestimentos asfálticos; patologia de pavimentos. 


\section{INTRODUÇÃO}

Dados do Departamento Nacional de Infraestrutura de Transportes (DNIT) e de uma pesquisa da Conferência Nacional de Transportes (CNT), ambos a partir de 2018, 60\% de todas as cargas no território e $57 \%$ das rodovias brasileiras apresentam algum tipo de manifestação patológica, o que refletem na cadeia produtiva do país.

Segundo o DNIT/IPR 720 (2006), "os pavimentos são concebidos para durarem um determinado período". O período compreendido entre o inicial e o final é denominado ciclo de vida do pavimento. Caso sejam realizadas manutenções periódicas o clico de vida poderá ser prolongado, reduzindo os custos operacionais da rodovia. Ainda de acordo com o DNIT, os pavimentos betuminosos apresentam tendência ao trincamento, devido a sua forma de atuação flexível sobre a base composta por solo e brita. A repetição de cargas, a qual se dá pela passagem de veículos, geram ciclos de fadiga, os quais ocasionam fissuramentos, e posteriormente as trincas.

Uma das técnicas empregadas na manutenção periódica, desde que o pavimento não apresente danos estruturais, é o microrrevestimento asfáltico, o qual pode ser seguido de uma remoção superficial de parte do revestimento desgastado ou não. Os microrrevestimentos são comumente aplicados na revitalização de camadas rolantes em estradas brasileiras. A norma técnica de referência, de acordo com o DNIT é a 035/2005-ES - Pavimentos flexíveis - Microrrevestimento asfáltico a frio com emulsão modificada por polímero - Especificação de serviço.

O desenvolvimento de novos materiais tecnológicos e sua aplicação em revestimentos asfálticos podem minimizar muitas das patologias atuais e reduzir custos com reparos futuros. A introdução do óxido de grafeno (GO) na matriz asfáltica, o qual atua como agente aglutinador, resultando na melhora das propriedades do revestimento asfáltico.

De acordo com SILVA (2017). “O termo "grafeno" não é novo e foi usado pela primeira vez em 1987, mas a definição oficial foi dada pela International Union of Pure and Applied Chemistry (IUPAC) em 1994”. Ainda segundo SILVA (2017) o grafeno é o nanomaterial mais pesquisado no mundo atualmente, devido as suas propriedades, as quais produzem melhorias nas propriedades dos demais materiais, entretanto seu custo de produção ainda é elevado, devido ao fato da produção em escala industrial estar em fase de implantação.

O grafeno possui espessura de um átomo, o seu derivado óxido de grafeno possui entre 3 a 20 vezes a espessura de um átomo, com custo de produção inferior ao do grafeno.

O presente estudo, por meio de revisão bibliográfica, tem por objetivo verificar a influência da utilização do óxido de grafeno nas propriedades do pavimento asfáltico.

Para o desenvolvimento da pesquisa, foi utilizada a técnica de Benchmarking, trata-se de uma metodologia utilizada para comparar os processos e as métricas de desempenho de um negócio, neste trabalho é analisado o resultado de pesquisas bibliográficas, sendo um comparativo entre as pesquisas realizadas, para identificar os resultados e sua empregabilidade, descobrindo assim, oportunidades de melhorias, no caso específico deste trabalho, o objetivo é verificar os resultados de aplicações e a possibilidade de utilização específica na camada de revitalização.

No primeiro capítulo é realizada a introdução e o objetivo do trabalho. No segundo capítulo é apresentada a revisão bibliográfica, com os conceitos empregados neste trabalho, referentes a Patologia de asfaltos, Microrrevestimento asfáltico, Grafeno, Óxido de Grafeno. No terceiro capítulo é realizada a exposição da Metodologia, a onde é apresentada as principais propriedades relatadas na pesquisa bibliográfica utilizando o óxido de grafeno em asfaltos. Por fim no quinto capítulo a conclusão do trabalho. 


\section{REVISÃO BIBLIOGRÁFICA}

\subsection{Patologia de Asfaltos}

As patologias presentes nos asfaltos podem ser classificadas em dois grupos. O primeiro de ordem estrutural, os quais tem sua origem em erros provenientes de projetos, falhas de material e de execução. O segundo grupo é de ordem funcional, devido ao desgaste natural devido a fadiga, proveniente do ciclo repetido de cargas durante seu uso e das intempéries.

Neste trabalho será abordado o segundo grupo de patologias, as quais são possíveis de serem corrigidas através do emprego do microrrevestimento asfáltico.

tendo como fonte de referência a norma do DNIT 005/2003 - Defeitos nos pavimentos flexíveis e semirígidos Terminologia.

De acordo com (Bernucci et. al., 2008), “as fendas são aberturas na superfície asfáltica e podem ser classificadas como fissuras, quando a abertura é perceptível a olho nu apenas à distância inferior a 1,5m, ou como trincas, quando a abertura é superior à da fissura".

Segundo a norma do DNIT 005/2003 as fissuras não apresentam danos funcionais ao pavimento. Pela classificação da citada norma as trincas podem ser:

- Trinca isolada, sendo subdividas em: transversal; longitudinal e de retração;

- Trinca interligada, subdivididas em: tipo "Couro de Jacaré" e tipo "Bloco".

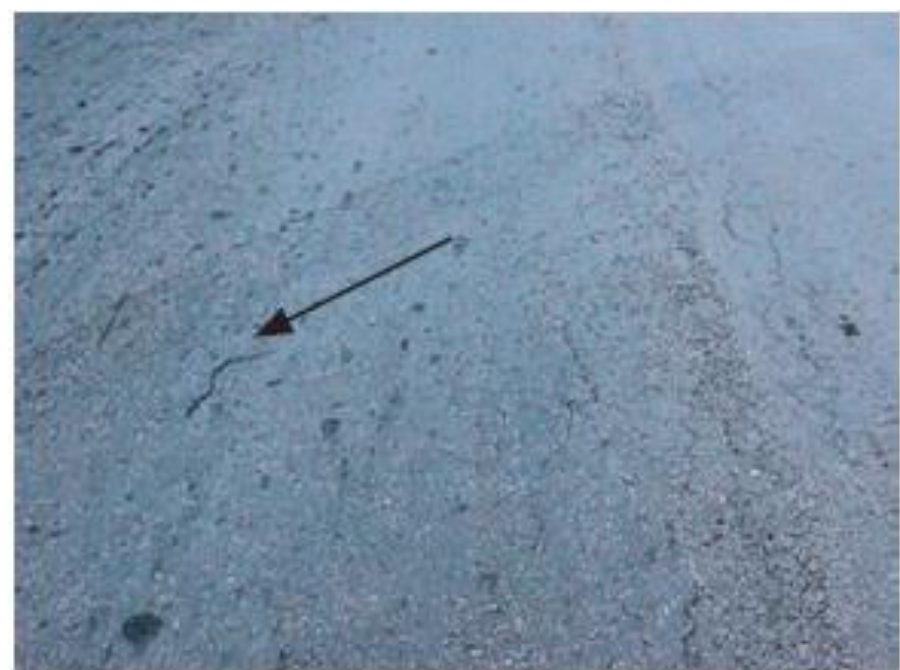

(a)

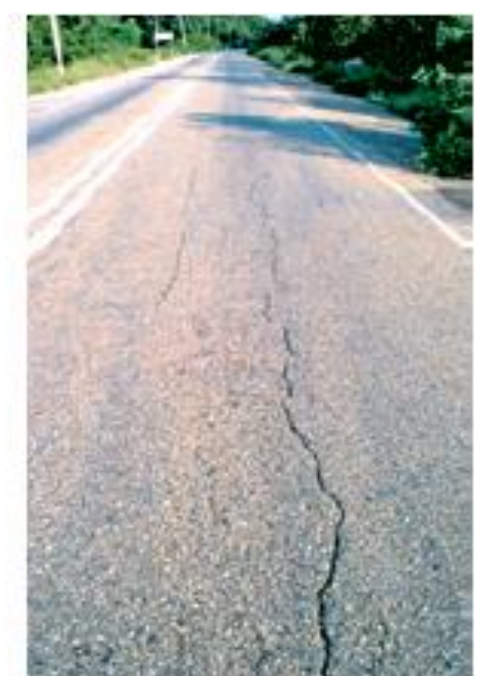

(b)

Figura 1. Exemplos de patologias em pavimentos, (a) Trinca isolada curta longitudinal e (b) Trincas longitudinais longas (Bernucci et. al., 2008).

\subsection{Microrrevestimento asfáltico}

Segundo (Bernucci et. al., 2008), o microrrevestimento asfáltico pode ser considerada evolução das lamas asfálticas, pois usa o mesmo princípio e concepção, contudo a adição de polímeros, permite a modificação das suas propriedades e por consequência a ampliação da sua vida útil. Sua aplicação é restrita a patologias de ordem funcional, com o objetivo de restaurar a capacidade de tráfego do pavimento, realizar o selamento da superfície e o prolongamento da vida útil do pavimento.

\subsection{Grafeno}

O grafeno foi descoberto em 2004, pelos pesquisadores Andre Geim e Konstantin Novoselov, pertencentes a Universidade de Manchester, como resultado do trabalho receberam o prêmio Nobel de Física no ano de 2010. Utilizando o grafite como matéria prima e fita adesiva, os pesquisadores obtiveram êxito em isolar uma única camada plana de átomos de carbono, com formato hexagonal. 
O grafeno é exatamente uma camada de carbono de grafite. Os átomos de carbono são dispostos em uma estrutura de favo de mel (S. Nazarpour e S. R.Waite, 2016).

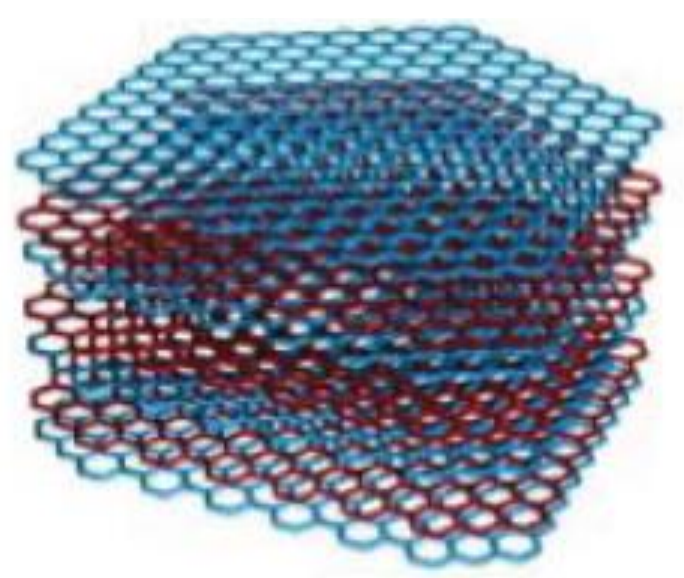

Figura 2. Representação gráfica da estrutura química do grafeno empilhado em camadas (S. Nazarpour e S. R.Waite, 2016).

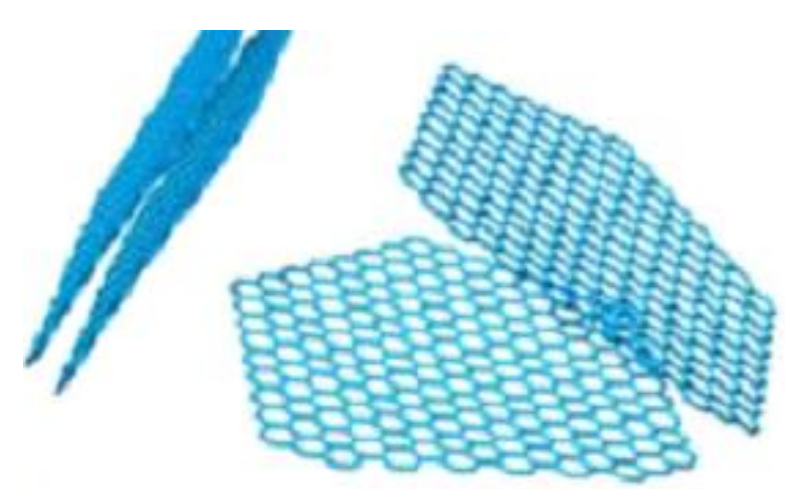

Figura 3. Representação gráfica da estrutura química do grafeno duas camadas isoladas (S. Nazarpour e S. R.Waite, 2016).

\section{4. Óxido de Grafeno}

O óxido de grafeno (GO) é obtido através do Método Modificado de Hummer, a partir da oxidação do grafite. O método se divide em três etapas distintas: oxidação, purificação e esfoliação. A oxidação é obtida através do uso dos seguintes agentes oxidantes concentrados: H2SO4, KMnO4 e H2O2. Conforme descrito por (Zhao et al., 2016) o método consiste em: "Após a oxidação, os produtos são centrifugados e depois esfoliados com o auxílio de vibração ultrassônica para obter a solução aquosa GO uniformemente dispersa". O resultado do processo é a formação de 3 a 20 camadas de grafeno agrupadas. 


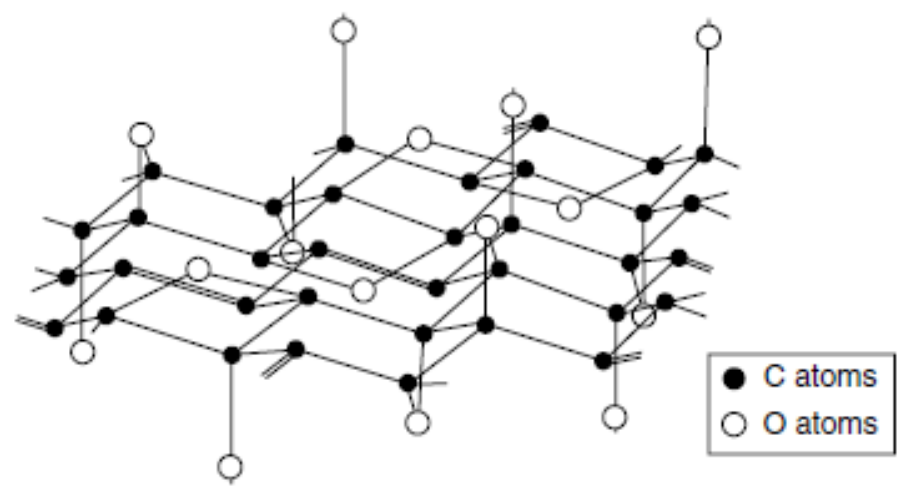

Figura 4. O modelo estrutural de acordo com Ruess, o modelo mostra claramente as duplas ligações deixadas não oxidadas (A. M. Dimiev e S. Eigler, 2017).

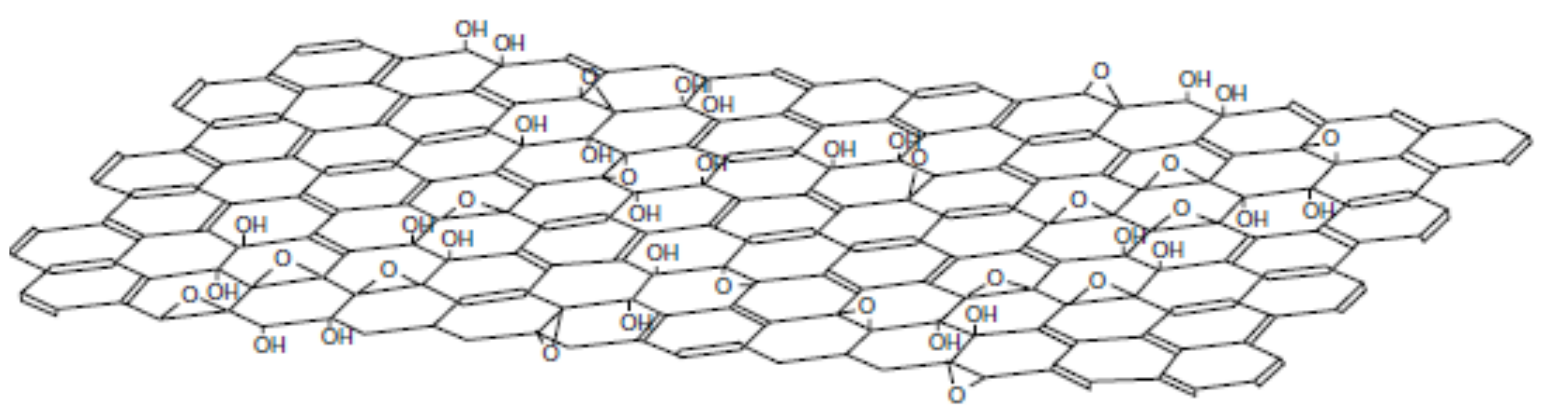

Figura 5. Modelo de Lerf-Klinowski da estrutura GO (A. M. Dimiev e S. Eigler, 2017).

Segundo (A. M. Dimiev e S. Eigler, 2017): “O óxido de grafeno (GO) é um material bidimensional (2D) derivado da matriz do grafeno introduzindo várias funcionalidades do oxigênio. Os átomos de oxigênio são covalentemente ligados a átomos de carbono, convertendo-os do estado sp2-hibridizado no grafeno-mãe no estado sp3-hibridizado".

\section{METODOLOGIA}

\subsection{Seleção de artigos}

Para o desenvolvimento desta revisão bibliográfica foram identificados 412 artigos, com os seguintes parâmetros de referência para a pesquisa:

3.1.1. Seleção por data de ocorrência - como critério foi estabelecida a ocorrência em pesquisas com data de publicação a partir de 2010. Devido a utilização do grafeno como compósito material ser recente.

3.1.2. Pesquisa por palavras-chave - com pesquisa inicial na base CAPES, foram adotadas as palavras ou expressões:

3.1.3. Combinação de palavras-chaves - considerando a palavra grafeno (a) e a palavra (d) foram associadas as demais palavras e expressões listadas de (a) a (i) para a obtenção dos resultados.

3.1.4. Aderência do título com o objetivo de pesquisa - a combinação entre grafeno e asfalto apresentou várias ocorrências relacionadas a pesquisas de supercondutores, as quais foram desconsideradas, pois não apresenta aderência ao tema pesquisado.

3.1.5. Leitura do Abstract - Após a primeira seleção foi realizada a leitura prévia dos resumos de cada artigo, a aderência aos objetivos da pesquisa foi definida como critério de seleção.

3.1.6. Leitura do artigo - como segundo critério de refinamento da pesquisa, foi adicionada a leitura das conclusões obtidas nos artigos.

3.1.7. Aderência relevante ao tema - artigos selecionados para desenvolvimento da análise 
comparativa de resultados.

Baseado na técnica descrita por (KESSLER,1963), sobre acoplamento de artigos, a seleção com a restrição de datas e utilizando a combinação descrita nos critérios, resultou na de ocorrência de 412 artigos, na data de 19/01/2019. Aplicando critérios de aderência ao tema pesquisado, verificando o título e as palavras-chave, foram selecionados 178 artigos, os quais se mantinham dentro dos objetivos pesquisados. Foi realizada a leitura dos artigos selecionados, restando na base de dados 42 artigos, os quais foram novamente submetidos aos critérios de relevância. Desta forma restaram 10 artigos os quais compõem a base de pesquisa para a apresentação dos resultados obtidos. Conforme apresentado na Figura 6, as quantidades de publicações dentro do objetivo de pesquisa apresentam um baixo percentual, 2,0\% de ocorrência de uma base de 412 ocorrências.

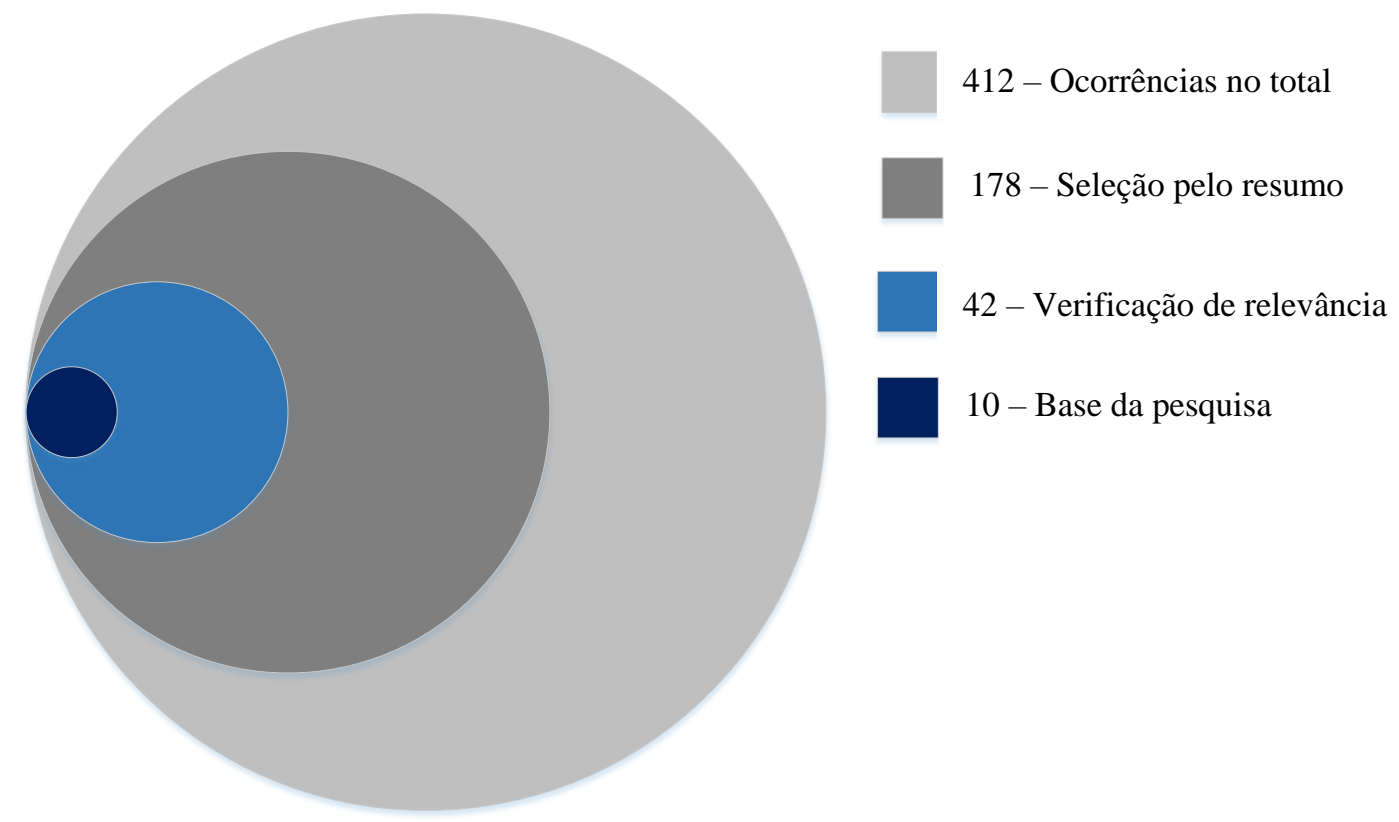

Figura 6. Representação gráfica - Artigos pesquisados.

\subsection{Propriedades identificadas nos resultados da pesquisa bibliográfica}

De acordo com (Bernucci et. al., 2008): “Todas as propriedades físicas do asfalto estão associadas à sua temperatura. $O$ modelo estrutural do ligante como uma dispersão de moléculas polares em meio não-polar ajuda a entender o efeito da temperatura nos ligantes asfálticos". A norma ABNT NBR 6576/98, assim como a norma europeia EM 12591/2000, apresenta ensaios correlacionando a temperatura com a viscosidade. Considerando a vida útil dos pavimentos a resiliência em relação ao ciclo de fadigas, as pesquisas contemplaram os resultados relativos à resistência a fadiga. A incorporação de água no asfalto após a utilização do óxido de grafeno foi considerada, devido a possibilidade de redução da sua oxidação e do seu envelhecimento. Todas as análises foram realizadas considerando o teor de óxido de grafeno adotado na composição, afim de descrever quais os percentuais indicados para a obtenção dos resultados.

\subsubsection{Resistência a fadiga}

O aumento da resistência entre os ligantes proporcionou uma maior resistência ao ciclo de fadiga, conforme demostrado pela pesquisa realizada por (Jing Li et al, 2018): "os percentuais utilizados entre 0,02 a 0,08\% apresentaram melhorias, aumentando a aglutinação entre as partículas, comparada a mistura original.

$\mathrm{Na}$ pesquisa foram adotados dois tipos de compostos asfálticos, com a adição de polímeros e sem a adição de polímeros. Os compostos apresentados na figura 6 identificados pelas siglas: GO (óxido de 
grafeno); PS (poliestireno); SBS (estireno butadieno estireno); PS-GNPs (nano plaquetas de óxido de grafeno combinadas com poliestireno); GNPs (nano plaquetas de óxido de grafeno).

A viscosidade do ligante foi medida nas temperaturas de $135^{\circ} \mathrm{C}, 165^{\circ} \mathrm{C}$ e $175^{\circ} \mathrm{C}$, através do emprego do viscosímetro de Brookfield de acordo com a norma T0625-2011(JING LI et al, 2018).

Os resultados apresentados, em comparação as amostras com sem adição do óxido de grafeno mostraram aumento na resistência a fadiga.

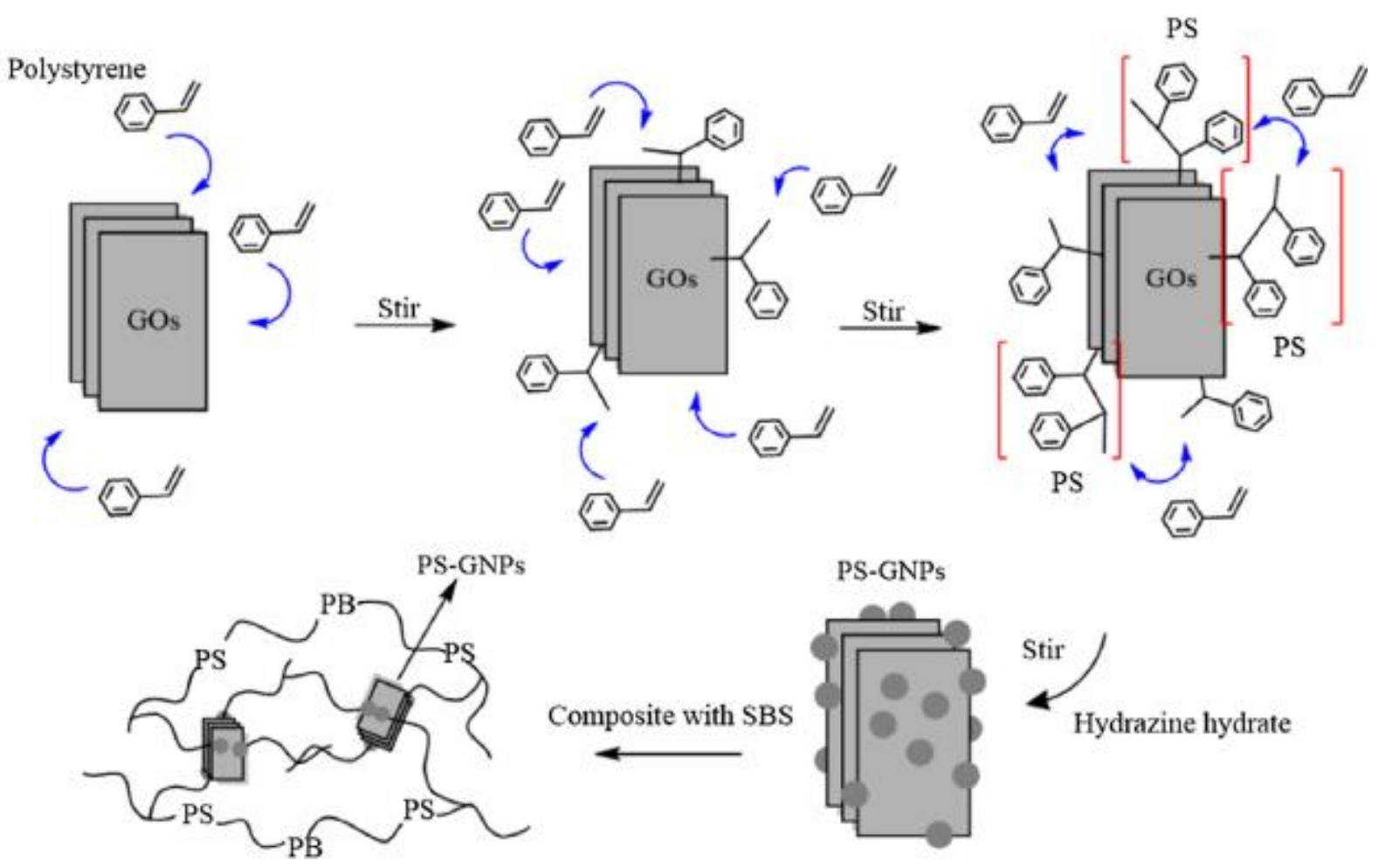

Figura 7. Representação esquemática do mecanismo de síntese do aglutinante incorporado por GNPsPS (Jing Li et al, 2018).
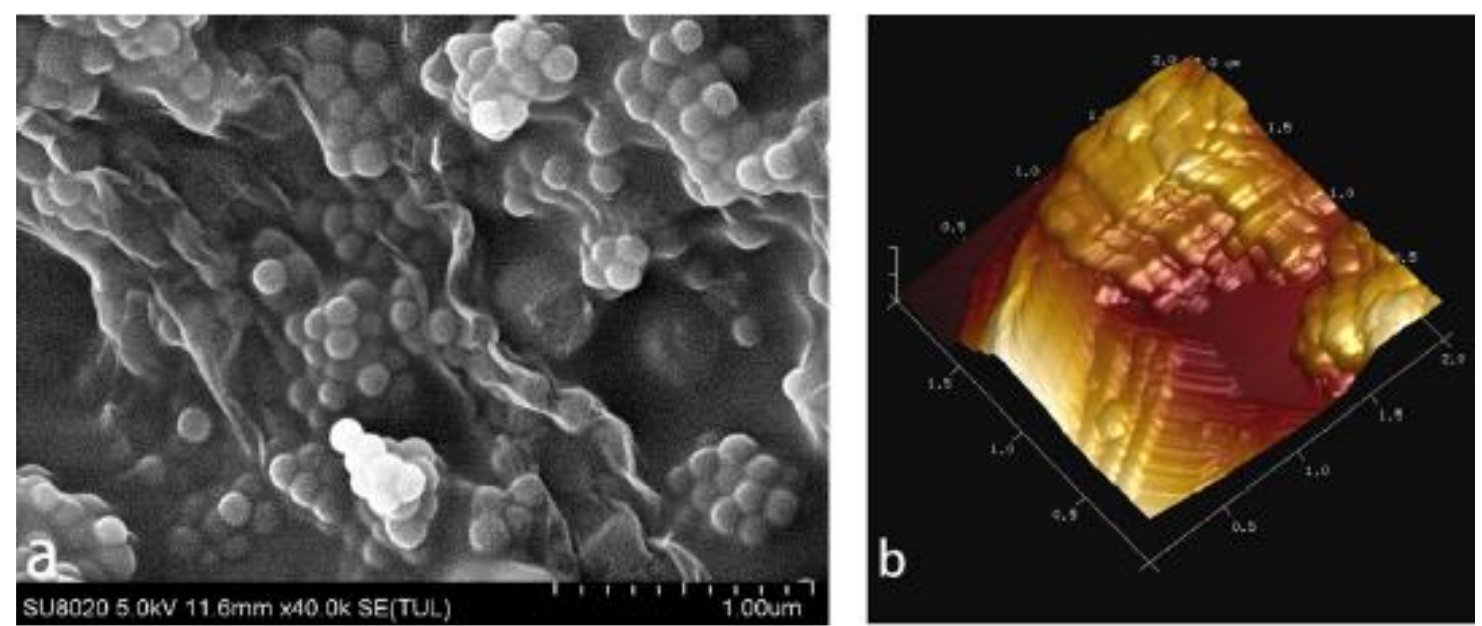

Figura 8. Imagens (a) MEV (Microscopia de Varredura Eletrônica) e (b) MFA(Microscopia de Força Atômica) de GNPs-PS (Jing Li et al, 2018).

Conforme apresentada na pesquisa de (Kefei Liu et al, 2018) a utilização do óxido de grafeno aumentou a capacidade de deformação do asfalto, conforme é apresentado no gráfico da figura 9 e figura 10 . O gráfico demostra a relação das amostras testas para os ciclos típicos de fluência e de recuperação no 
qual foram empregados os carregamentos de teste com $0,1 \mathrm{kPa}$ e com 3,2 $\mathrm{kPa}$. As amostras utilizadas: HMAB-1 (100,00\% mistura de asfalto a quente - amostra de controle) ; HMAB-2 (99,5\% mistura de asfalto a quente $+0,05 \%$ de GO); WMAB- $1(96,95 \%$ mistura de asfalto a quente com aditivo aglutinante $+3,00 \%$ Sasobit $+0,05 \%$ de GO); WMAB-2(94,95\% mistura de asfalto a quente com aditivo aglutinante $+5,00 \%$ de óleo de cozinha usado $+0,05 \%$ de GO); WMAB-3 (91,95\% mistura de asfalto a quente com aditivo aglutinante $+5,00 \%$ de óleo de cozinha usado+ $0,05 \%$ de GO $+3,00 \%$ Sasobit). Os valores de entre recuperação e carregamento do asfalto modificado GO + WCO (WMAB-2) foram $33 \%$ vezes maiores e $48 \%$ menores que os do asfalto de controle, respectivamente (Kefei Liu et al, 2018).

Carregamento aplicado: $0,1 \mathrm{kPa}$

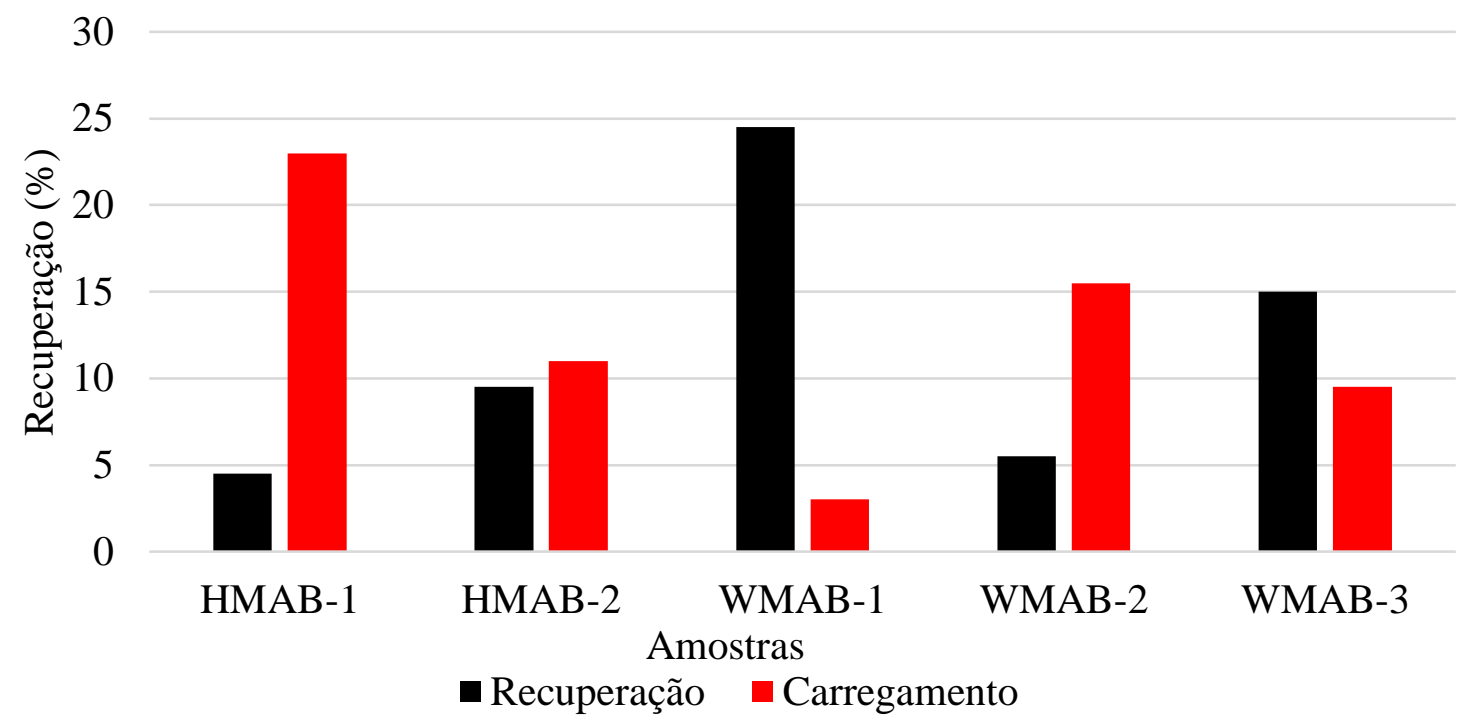

Figura 9. Gráfico do ciclo típico de fluência e recuperação para os HMABs e WMABs a com carga aplicada de 0,10 kPa, adaptado (Kefei Liu et al, 2018).

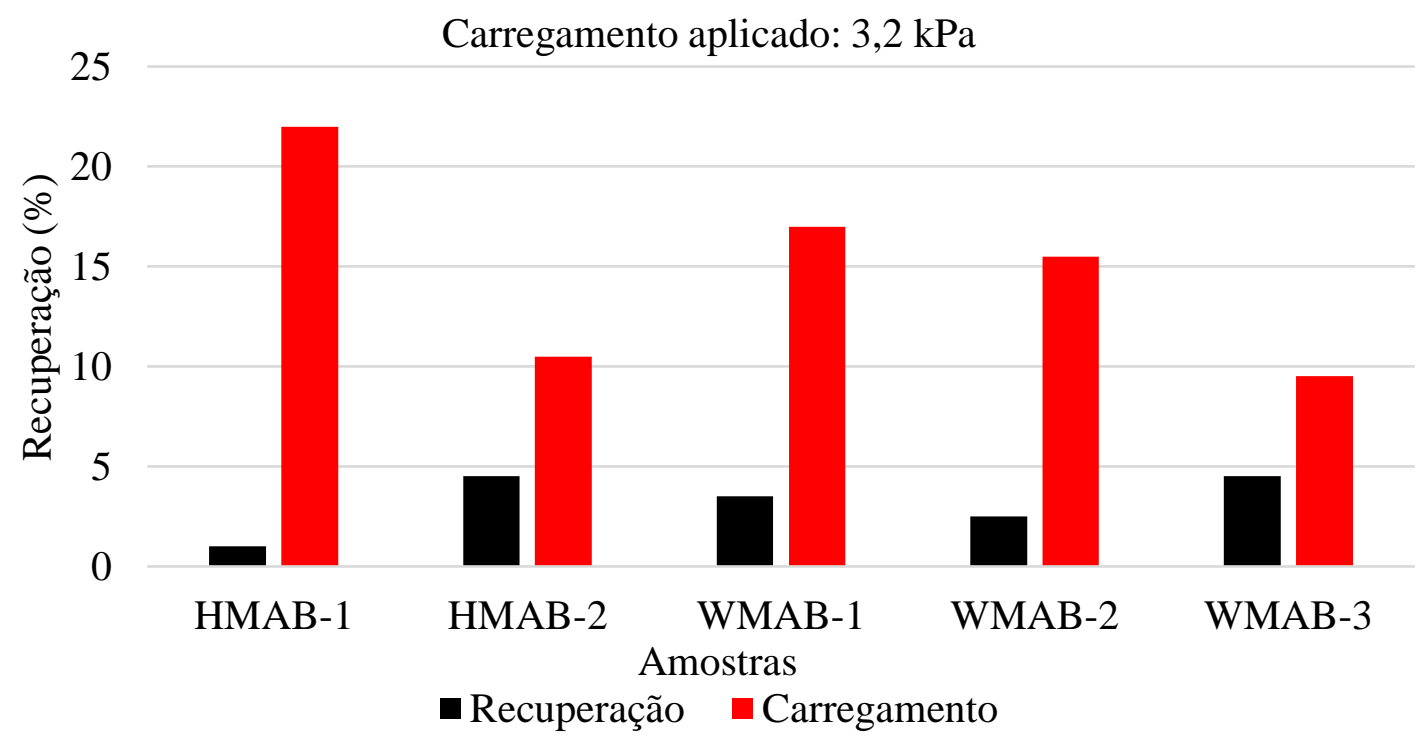

Figura 10. Gráfico do ciclo típico de fluência e recuperação para os HMABs e WMABs a com carga aplicada de 3,20 kPa, adaptado (Kefei Liu et al, 2018). 
Segundo a pesquisa realizada por (Brcic, 2016) a adição do grafeno aumentou do módulo de elasticidade (E) da mistura asfáltica e a recuperação da elasticidade. Na pesquisa foram utilizadas adições entre $1,00 \%$ e 2,00\% de grafeno, resultando em variação no módulo de elasticidade entre 3,00 $\mathrm{MPa}$ e 17,9 MPa, proporcional ao teor adicionado.

De acordo com (Jing et al, 2018): "A resistência à temperatura e as propriedades mecânicas dos asfaltos foram analisadas ensaios de reologia dinâmica de cisalhamento (DSR) e de recuperação por fluência sob tensão múltipla (MSCR), que revelaram que uma quantidade ótima de GOs-SH (0,02\%) pode efetivamente melhorar a baixa temperatura e desempenho anti fissuras do asfalto $S B S$ ”.

\subsubsection{Resistência a incorporação de água}

Conforme indicado na figura 11, a incorporação do óxido de grafeno melhorou as propriedades de resistência a incorporação de água, os teores empregados no asfalto modificado: 0,02\% a 0,08\% de GO (Jing Li et al, 2018).

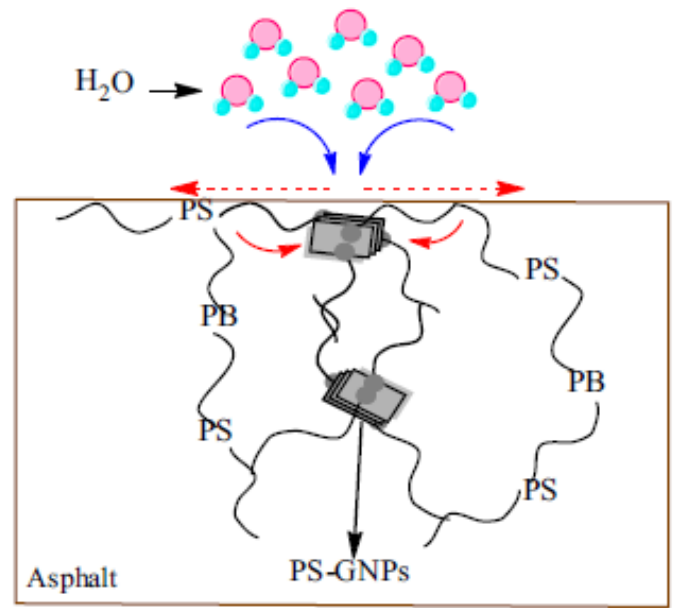

Figura 12. Ilustração do mecanismo de bloqueio da entrada de água no asfalto modificado PS-GNPs (nano plaquetas de óxido de grafeno combinadas com poliestireno) (Jing Li et al, 2018).

\subsubsection{Deformação plástica}

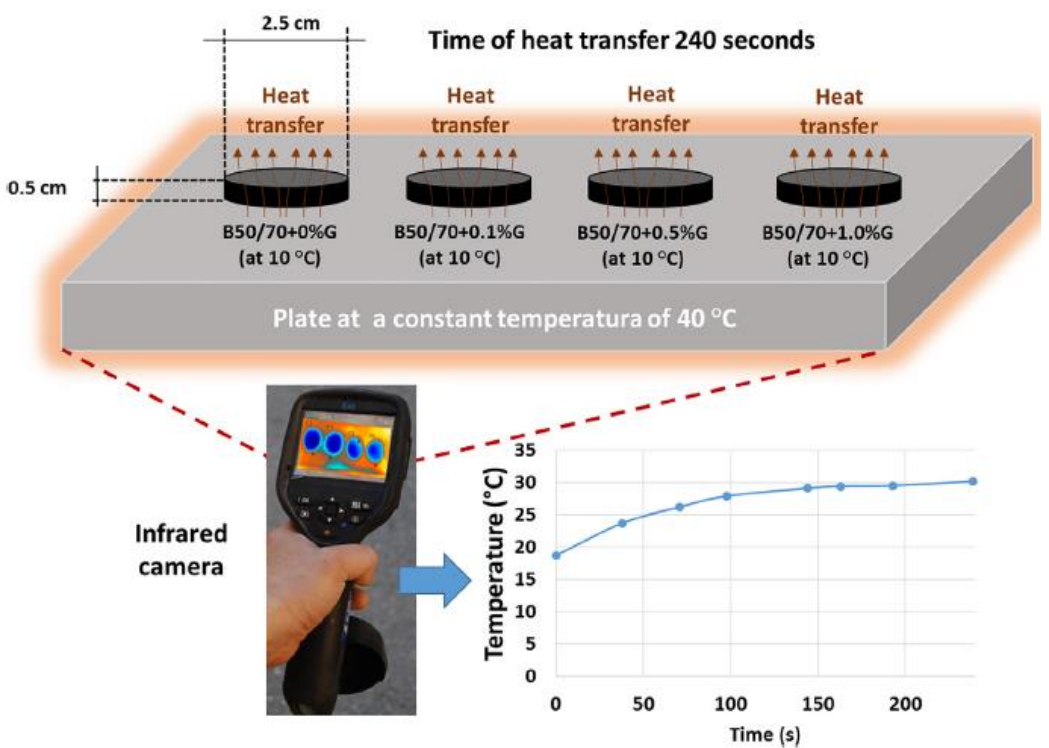

Figura 13. Esboço do experimento para determinar a capacidade de aquecimento dos ligantes de asfalto, é realizada a leitura das variações de temperatura x tempo (Navarro, 2018). 
A pesquisa sobre a adição de grafeno com as proporções de $0,1 \%, 0,5 \%, 1,0 \%$ e 3,0\% em peso de asfalto. Foram observados resultados relativos à resistência a deformações plásticas a altas temperaturas. Conforme é apresentado o esquema de ensaio na figura 13. A adição de grafeno resulta em aumento da temperatura do ligante, tendo como consequência o aumento do desempenho das deformações plásticas (Navarro, 2018).

De acordo com (Navarro, 2018) uma proporção elevada de grafeno na composição do asfalto poderá torna-lo frágil.

Ao utilizar percentuais variando entre $0,50 \%$ e $3,00 \%$ de grafeno, foi verificada uma variação de temperatura de trabalho inicial de $64^{\circ} \mathrm{C}$ para $76^{\circ} \mathrm{C}$, refletindo na melhoria da deformação plástica e da resistência do asfalto (Amin, 2016).

\subsubsection{Viscosidade}

Segundo Kefei Liu (2018): "Isso indica que a adição de 0,05\% em peso de GO obviamente aumentou a viscosidade do asfalto". Conforme é apresentado na figura 14, foi comparada a viscosidade entre das cinco amostras tipos de asfalto (HMAB-1, HMAB-2, WMAB-1, WMAB-2 e WMAB-3), com e sem a adição do óxido de grafeno.

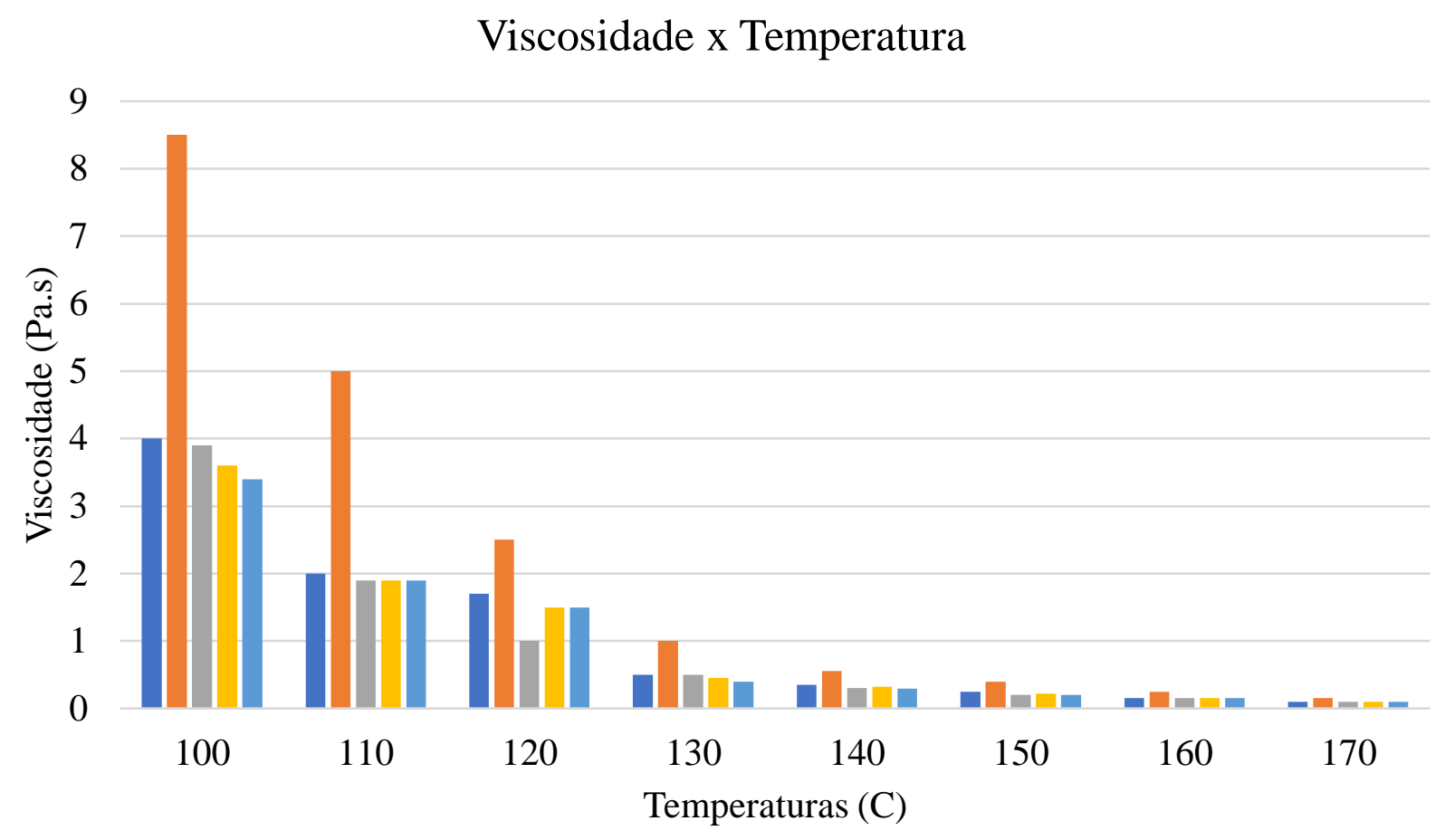

"HMAB-1 $\square$ HMAB-2 $\square$ WMAB-1 $\square$ WMAB-2 $\square$ WMAB-3

Figura 14. Desempenho da Viscosidade x Temperaturas das amostras, adaptado (Kefei Liu et al, 2018).

Quando comparadas as amostras HMAB-1 e HMAB-2 (com adição de 0,05\% de GO), fica evidenciado o melhor desempenho do óxido de grafeno (Kefei Liu et al, 2018).

Segundo (Kefei Liu et al, 2018): "Esses resultados sugerem que a adição de $0,05 \%$ em peso de GO pode aumentar o nível de tráfego no pavimento asfáltico do nível padrão ao pesado, e mais de $3 \% \mathrm{em}$ peso".

De acordo com (Jing Li et al, 2018): “A resistência à temperatura e as propriedades mecânicas dos asfaltos foram analisadas ensaios de reologia dinâmica de cisalhamento (DSR) e de recuperação por 
fluência sob tensão múltipla (MSCR), que revelaram que uma quantidade ótima de GOs-SH $(0,02 \%)$ pode efetivamente melhorar a baixa temperatura e desempenho anti fissuras do asfalto SBS".

O emprego do óxido de grafeno, dosagem entre 1,0\% a 3,0\% na mistura asfáltica, proporcionou melhora na mistura asfáltica, através da comparação entre viscosidade versus temperatura (Y. Li et al, 2018). Ainda segundo (Y. Li et al, 2018): "Os resultados indicaram que quando a taxa de dosagem GO de 3\% utilizado, o incremento de viscosidade máxima de 12,5\% (e 15,0\%) e o incremento de viscosidade mínimo de 5,4\% (e 4,1\%) obtido".

O estudo realizado por (Kefei Liu et al, 2018), utilizando os seguintes teores de óxido de grafeno nas dosagens de asfalto com SBS modificado: 0,00\% A0 (amostra de controle); A0.02 (amostra + 0,02\% GO); A0.05 (amostra + 0,05\% GO); A0.10 (amostra + 0,10\% GO); A0.20 (amostra + 0,20\% GO) e $1,00 \%$ A1 (amostra $+1,00 \%$ GO). Conforme apresentado na figura 15, o desempenho da viscosidade $\mathrm{x}$ temperatura demostrou maior eficiência na dosagem de $0,05 \%$ de óxido de grafeno.

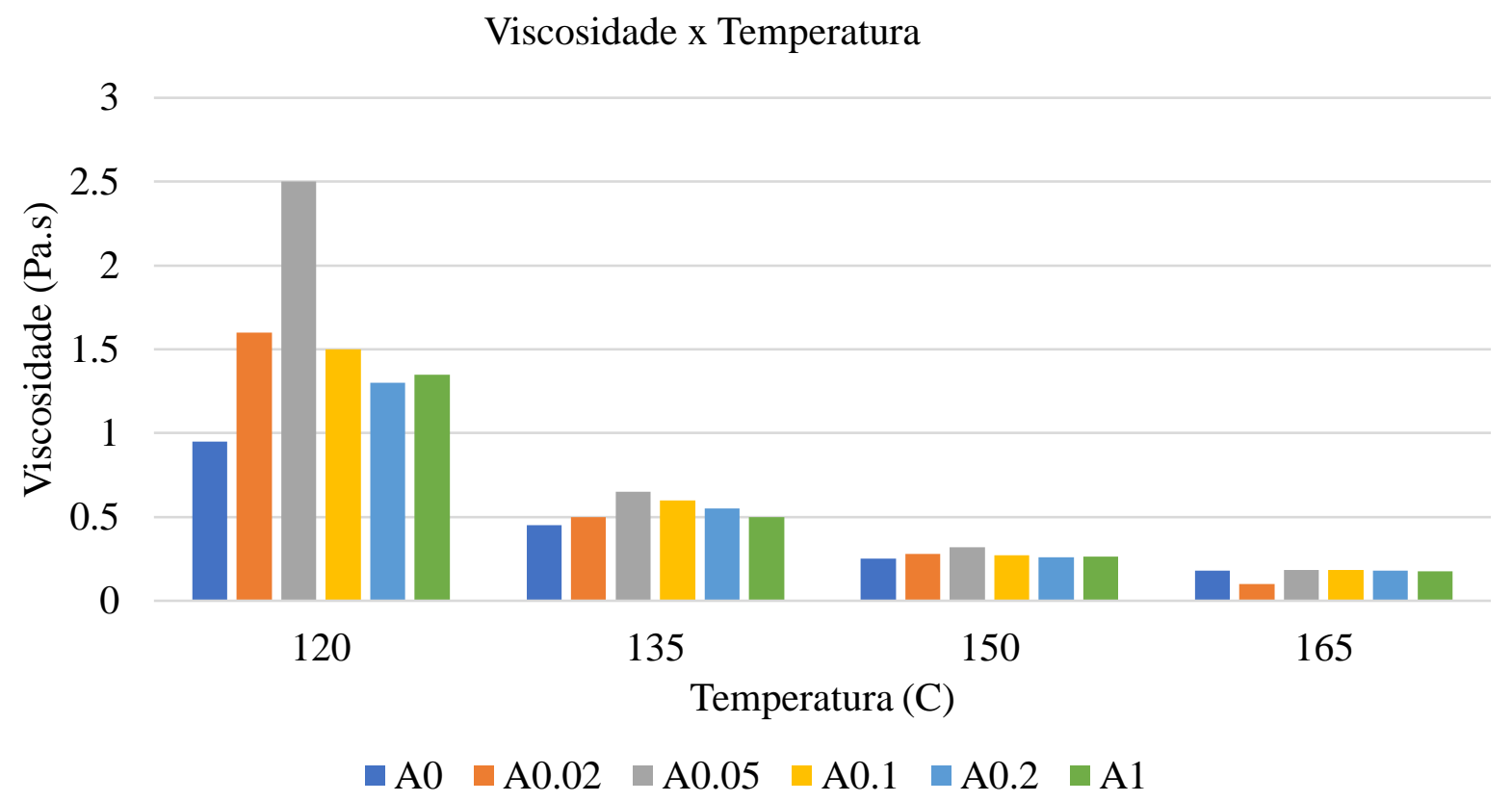

Figura 15 - Gráfico comparativo entre viscosidade x temperatura, devido a influência da adição de GO nas amostras, adaptado (Kefei Liu et al, 2018).

Segundo KEFEI (2018): "O GO pode rapidamente adsorver moléculas grandes (como colóides e asfaltenos) no ligante de asfalto, aumentando significativamente este material em termos da viscosidade da temperatura de pavimentação, resistência à deformação permanente e função elástica".

\section{CONCLUSÃO}

As pesquisas bibliométricas comprovam a viabilidade da utilização do óxido de grafeno adicionado a mistura asfáltica, os teores identificados nas pesquisas consultadas variam de $0,05 \%$ a $3,0 \%$ de adição sobre o peso do asfalto. Adições inferiores a $0,5 \%$ não demostraram eficiência para todas as propriedades, sendo o teor ideal indicado nas pesquisas de 2,0\%, as principais propriedades melhoradas são:

\subsection{Viscosidade}

Segundo (Y. Li et al, 2018) ao utilizar o percentual de 3,0\% de óxido de grafeno a viscosidade teve ganho entre $5,4 \%$ a $12,5 \%$. 


\subsection{Variação de temperatura}

Segundo (AMIN, 2016), adicionar 3,0\% de óxido de grafeno a mistura asfáltica, aumentou a alta temperatura de $64^{\circ} \mathrm{C}$ para $76^{\circ} \mathrm{C}$.

\subsection{Resistência a deformação}

De acordo com (Jing Li et al, 2018), a redução do fissuramento em baixa temperatura. Segundo KEFEI (2018), o óxido de grafeno, aumenta a resistência a deformação permanente e função elástica da mistura asfáltica.

\subsection{Resistência aos ciclos de fadiga}

Segundo (Kefei et al, 2018). A adição de $0,05 \%$ em peso de GO pode aumentar o nível de tráfego no pavimento asfáltico do nível padrão ao pesado, e mais de 3,0\% em peso. Segundo (Jing Li et al, 2018), os percentuais utilizados entre 0,02 a $0,08 \%$ apresentaram melhorias, aumentando a aglutinação entre as partículas, comparada a mistura original.

Para aplicação no Brasil é necessário definir qual seria a região, pois a função variação climática, a viscosidade indicada, o teor de hidrocarbonetos da emulsão asfáltica e a resistência a deformação, irão influenciar no teor de óxido de grafeno ideal a ser adotado. Entretanto é inegável, conforme as pesquisas, os benefícios em razão da utilização do óxido de grafeno na mistura asfáltica.

\section{REFERENCIAS}

Amin I. et. all. (2016), Laboratory evaluation of asphalt binder modified with carbon nanotubes for Egyptian climate. Construction and Building Materials $121 \quad 361-372 \quad$ http://dx.doi.org/10.1016/j.conbuildmat.2016.05.168

Bernucci L. B. et. all. (2006). Pavimentação Asfáltica - Formação Básica para engenheiros. Rio de Janeiro: PETROBRAS: ABEDA, CDD 625.85.

Brcic H. (2016), Investigation of the graphene oxide and asphalt interaction and its effect on asphalt pavement performance. Norwegian University of Science and Technology. Department of Civil ad Transport Engineering.

Dimiev A. M. (2017), Graphene Oxide: fundamentals and applications. John Wiley \& Sons, Inc., ISBN 9781119069430.

DNIT (2003), NORMA DNIT 005/2003 - TER - Defeitos nos pavimentos flexíveis e semi-rígidos Terminologia.

DNIT (2005), NORMA DNIT 035/2005 - ES Pavimentos flexíveis - Micro revestimento asfáltico a frio com emulsão modificada por polímero - Especificação de serviço.

Han M. et. all. (2018), Studies on the secondary modification of SBS modified asphalt by the application of octadecyl amine grafted graphene nano platelets as modifier. Diamond \& Related Materials 89140 150 - https://doi.org/10.1016/j.diamond.2018.08.011

Jing Li et. all. (2018), Comparative analysis, road performance and mechanism of modification of polystyrene graphene nanoplatelets (PS-GNPs) and octadecyl aminegraphene nanoplatelets (ODAGNPs) modified SBS incorporated asphalt binders. Construction and Building Materials 193 501-517 - https://doi.org/10.1016/j.conbuildmat.2018.10.210

Jing L. et. all. (2018), Preparation and Properties of SBS-g-GOs-Modified Asphalt Based on a Thiolene Click Reaction in a Bituminous Environment. Polymers, 10, 1264 www.mdpi.com/journal/polymers - https://doi:10.3390/polym10111264

Yao H. et. all. (2017), Property Analysis of Exfoliated Graphite Nanoplatelets Modified Asphalt Model Using Molecular Dynamics (MD) Method, Appl. Sci. 7, 43 - www.mdpi.com/journal/applsci https://doi:10.3390/app7010043 
Yuanyuan L. et. all. (2018), Investigation of the graphene oxide and asphalt interaction and its effect on asphalt pavement performance. Construction and Building Materials $165572-584$ https://doi.org/10.1016/j.conbuildmat.2018.01.068

Kefei L. et. all. (2018), Performance evaluation and modification mechanism analysis of asphalt binders modified by graphene oxide. Construction and Building Materials 163 880-889 https://doi.org/10.1016/j.conbuildmat.2017.12.171

Kefei L. et. all. (2018), Evaluation of mechanical performance and modification mechanism of asphalt modified with graphene oxide and warm mix additives. Journal of Cleaner Production 193 87e96 https://doi.org/10.1016/j.jclepro.2018.05.040

Kessler, M. M. (1963). Bibliographic coupling between scientific papers. American Documentation, v. 14, p. 10-25.

Kessler, M. M. (1965), Comparison of the results of bibliographic coupling and analytic subject indexing. American Documentation, v. 16, n.3, p. 223-233.

Nazarpour S. et. all. (2016), Graphene Technology from Laboratory to Fabrication. Wiley-VCH Verlag GmbH \& Co. Weinheim, Germany.

Moreno-Navarrro F. et. all. (2018), Mechanical and thermal properties of graphene modified asphalt binders. Construction and Building Materials $180 \quad 265-274$ https://doi.org/10.1016/j.conbuildmat.2018.05.259

Zhao, L. et. all. (2016), Investigation of the effectiveness of PG@GO on the reinforcement for cement composites. Construction and Building Materials 113, 470-478. 